\title{
A SATISFAÇÃO, REALIZAÇÃO E EXAUSTÃo dOS ENFERMEIROS EM PORTUGAL
}

\author{
Carla Cruz \\ Adjunt Professor in Superior Health School \\ Polytechnic Institute of Viseu - PhD \\ Paula Nelas \\ Adjunt Professor in Superior Health School \\ Polytechnic Institute of Viseu - PhD \\ Emília Coutinho \\ Adjunt Professor in Superior Health School \\ Polytechnic Institute of Viseu - PhD \\ Cláudia Chaves \\ Adjunt Professor in Superior Health School \\ Polytechnic Institute of Viseu - PhD \\ Odete Amaral \\ Adjunt Professor in Superior Health School \\ Polytechnic Institute of Viseu - PhD
}

Fecha de Recepción: 13 Marzo 2018

Fecha de Admisión: 10 Abril 2018

\section{RESUMO}

Contexto: As mudanças na carreira profissional e condições de trabalho implicam comprometimento da saúde física e mental, com prejuízo na satisfação, realização e exaustão dos enfermeiros portugueses.

Objetivo(s): Verificar se as variáveis sociodemográficas e profissionais e interferem na exaustão, satisfação e realização pessoal e profissional. Analisar se a satisfação profissional interfere na exaustão sentida pelos Enfermeiros portugueses.

Metodologia: Estudo de natureza quantitativa, descritivo, correlacional e analítico, retrospetivo e transversal. Amostra não probabilística, constituída por 534 enfermeiros portugueses $(23.8 \%$ homens e $76.2 \%$ mulheres, com idades entre os 21 e 57 anos. Instrumento de colheita de dados: questionário sociodemográfico e profissional; Escala de Satisfação e Realização; Questionário de Copenhagen Burnout Inventory.

0 tratamento de dados foi efetuado com o programa SPSS (Statistical Package for the Social Sciences) versão 24 para Windows.

Resultados: As variáveis: sexo, estado civil; idade; formação especializada; tempo de serviço; horas de trabalho semanal e modalidade; recompensas; tipo de instituição pública/privada, interfe- 


\section{A SATISFAÇÃO, REALIZAÇÃO E EXAUSTÃO dOS ENFERMEIROS EM PORTUGAL}

rem com a exaustão, satisfação e realização pessoal e profissional. Os enfermeiros pouco satisfeitos apresentam exaustão emocional e despersonalização, $(0 \mathrm{M}=347.60)$ e $(0 \mathrm{M}=299.79)$ respetivamente. A nível da realização pessoal prevalecem os que se manifestam muito satisfeitos (OM=301.40). Na totalidade da avaliação do nível de burnout, há um predomínio de enfermeiros pouco satisfeitos $(0 \mathrm{M}=327.05)$.

Conclusões: É urgente implementar medidas a nível da gestão e direção das instituições de saúde com o objetivo de diminuir a carga de trabalho, através da contratação de enfermeiros e outras medidas como o desenvolvimento de programas de intervenção sobre gestão do tempo, conflitos, relação interpessoal e comunicação; Estimulo ao reconhecimento do trabalho, valorização e delegação das competências e sistema de recompensas dos enfermeiros.

Palavras-Chave: burnout; enfermagem; Portugal; satisfação

\section{ABSTRACT}

Satisfaction, fulfillment and exhaustion in portuguese nurses. Background:

Changes the level of the professional career and working conditions, implies impairment of physical and mental health, with prejudice on satisfaction, fulfillment and exhaustion of nurses in Portugal.

Objectives: Verify if the socio-demographic and professional variables interfere with exhaustion, satisfaction, personal and professional fulfillment. Consider whether job satisfaction interfere with the exhaustion felt by nurses in Portugal.

Methodology: It is a quantitative, descriptive, correlational and analytical, retrospective and cross study, whose non-probabilistic sample consists of 534 Portuguese Nurses $(23.8 \%$ men and $76.2 \%$ women), aged between 21 and 57 years $(m=34.83)$. It was used as data collection the sociodemographic and professional questionnaire, Satisfaction and achievement Scale the Copenhagen Burnout Inventory and the Maslach Burnout Inventory. The data was processed with the SPSS program (Statistical Package for the Social Sciences) version 24 for Windows.

Results: The variables: sex, marital status; age; specialized training; time of service; weekly working hours and mode; rewards; public/private institution type, interfere with exhaustion, satisfaction, personal and professional fulfillment. Nurses who show emotional exhaustion and depersonalization are least satisfied ( $M W=347.60)$; $(0 M=299.79)$ respectively. In terms of personal achievement prevail manifested the ones who are very satisfied (MW $=301.40$ ). The entire assessment of burnout level, there is a predominance of little satisfied Nurses ( $M W=327.05)$.

Conclusion: In the light of the results obtained and the specificity of the work is urgent implement measures in the management and direction of the health institutions in the sense to lessen the workload by hiring nurses and other measures as the development of intervention programs on time management, conflict, interpersonal relationship and communication; Encourage the recognition of work, valuation and delegation of powers and system of rewards of nurses.

Keywords: burnout; nursing; Portugal; fulfillment

\section{INTRODUÇÃO}

0 Enfermeiro possui expectativas profissionais de ascensão na carreira articuladas com responsabilidades, direitos e deveres, condições de trabalho e renumeração adequada.

Face ao atual contexto político económico e social o panorama laboral nacional tem sofrido profundas modificações a nível da empregabilidade com alterações da carreira de enfermagem, que se reflectem na satisfação dos enfermeiros, facto que tem sido divulgado também nos diversos meios de comunicação social. 
A satisfação, um dos fatores que poderá interferir na qualidade do desempenho de uma profissão, é definida como um estado afetivo positivo, resultante da apreciação do emprego ou das expectativas ligadas ao emprego (Menezes, 2010). Este sentimento, quando desfavorável, pode comprometer a eficácia da prestação de cuidados e a saúde dos trabalhadores.

Associado aos fatores geradores de insatisfação os enfermeiros, ao cuidarem continuamente pessoas em situação de fragilidade física e/ou psicológica, estão mais predispostos a exaustão física, mental e social (Gomes (2009), Martins (2010) e Fonte (2011).

Ainda neste contexto, a depleção de energia pelo stress não aliviado, a falta de apoio e de relação social, os conflitos entre as expectativas e a realidade profissional pode implicar uma situação de Burnout (Conselho Internacional de Enfermeiros, 2011).

Face a esta realidade questionamo-nos se a satisfação profissional interfere na exaustão sentida pelos enfermeiros a exercer atividade profissional em Portugal e se as variáveis sociodemográficas e profissionais interferem na exaustão, satisfação e realização pessoal e profissional.

\section{METODOLOGIA}

Delineamos uma pesquisa de natureza quantitativa, descritiva, correlacional e analítica, retrospetiva e transversal. Utilizamos como instrumento de colheita de dados um questionário que permitiu a caracterização sociodemográfica e profissional da amostra, a Escala de Satisfação e Realização,Copenhagen Burnout Inventory e o Maslach Burnout Inventory (MBI). A colheita de dados foi realizada via online e os dados foram tratados com o programa SPSS (Statistical Package for the Social Sciences) versão 24.0.

\section{RESULTADOS}

Traçando um perfil sociodemográfico e profissional da amostra constatamos que os 534 enfermeiros portugueses a exercer atividade profissional em Portugal, apresentam uma idade mínima de 21 anos e máxima de 57 anos ( $X=34.83)$, tendo o grupo etário mais representativo (36.1\%) idades compreendidas entre os 30 e os 36 anos de idade.

Prevalecem as mulheres $(76.2 \%)$, com idade $\leq 29$ anos $(26.0 \%)$. No que se refere ao estado civil, $54.1 \%$ dos enfermeiros são casados ou vivem em união de facto. A nível da formação académica, $88.2 \%$ dos participantes possuem o grau académico de licenciatura.

Relativamente ao tempo total de exercício profissional, 46.4\% possuem entre 1-10 anos de exercício profissional, exercendo funções numa instituição de saúde pública $81.2 \%$. No que se refere ao regime de trabalho ou natureza do vínculo, 53.2\% enfermeiros, possuem contrato de trabalho por tempo indeterminado. A categoria atual é Enfermeiro (88.6\%), 38,8\% possuem Especialidade ou Pós-graduação, referindo exercer a atividade profissional no local de trabalho opção própria $(66.1 \%)$, manifestando-se satisfeitos $56 \%$. 0 tempo de deslocação de casa/trabalho é <30 minutos $(85.0 \%)$.

Em relação ao serviço atual onde os enfermeiros exercem funções, $28.1 \%$ exerce em especialidades médicas, medicina, reabilitação e cuidados continuados, $18.0 \%$ em cuidados intensivos, urgência, emergência e neonatologia, 16.7\% trabalham no bloco operatório, cirurgia, ortopedia e $15.9 \%$ em cuidados de saúde primários.

No que se refere ao tipo de horário praticado, $71.3 \%$ pratica um horário rotativo, $62.0 \%$ têm um horário de trabalho semanal de 35-40 horas e $62.5 \%$ refere que no último ano, o número de enfermeiros da sua equipa foi reduzido, sendo o número de enfermeiros diminuto faces às necessidades (80.1\%). Verificamos que $58.8 \%$ dos enfermeiros faz turnos suplementares/extra com regularidade, sobressaindo $36.7 \%$ de enfermeiros cujo número de horas acrescido ao seu horário mensal em 


\section{A SATISFAÇÃO, REALIZAÇÃO E EXAUSTÃO dOS ENFERMEIROS EM PORTUGAL}

média é de 8 a 16 horas. 56.0\% dos participantes afirmam ser recompensados em tempo disponível, pela realização de turnos suplementares/extra.

Quase a totalidade da amostra (83.0\%) admite que, se pudesse, recusava a realização de turnos suplementares/extra, independentemente da recompensa recebida. É expressivo o percentual de $89.3 \%$ dos enfermeiros que admitem não ter sido vítima de algum acidente de trabalho nos últimos seis meses e $95.7 \%$ consideram que a sobrecarga de trabalho os torna suscetíveis.

A maioria dos participantes $(82.4 \%)$ sente diminuição da concentração na realização das atividades diárias, referindo que os elementos da sua equipa, nos últimos seis meses, estão mais concentrados no trabalho individual do que no trabalho de equipa (74.0\%) e $61.6 \%$ admitem que interrompem e reiniciam a mesma atividade várias vezes.

No que respeita à forma como habitualmente 0 Enfermeiro chefe/gestor lida com as situaçõesproblema do serviço, nos últimos seis meses, $69.9 \%$, tem sentido mais dificuldade em lidar com os conflitos na equipa.

Quanto ao facto de já terem pensado em mudar de serviço nos últimos meses, a maioria $(62.9 \%)$ dos enfermeiros admitiram essa hipótese, fazendo parte dos planos de $22.1 \%$ emigrarem, contrariamente a $77.9 \%$.

Tendo em consideração a relação entre o burnout e as variáveis sociodemográficas e profissionais, verificamos que as enfermeiras manifestam mais exaustão emocional e realização, comparativamente aos enfermeiros, tendo resultado em diferenças estatisticamente significativas para a exaustão emocional. São os enfermeiros que manifestam mais despersonalização, no entanto sem significância estatística. No total do burnout, registamos uma ordenação média mais alta para as enfermeiras, apresentando maiores índices de burnout.

Os enfermeiros com idade igual ou superior aos 37 anos são os que se revelam mais exaustão emocional, resultando em diferenças estatisticamente significativas. Os enfermeiros com idade igual ou inferior aos 29 anos, apresentam valores mais elevados de despersonalização e os com idade igual ou superior a 37 anos apresentam valores mais baixos, com significância estatística. A nível da realização, as ordenações médias encontradas entre os enfermeiros mais novos e os mais velhos estão muito próximas, no entanto, é no grupo dos enfermeiros com idade igual ou inferior a 37 anos onde é encontrada relevância estatística.

Apresentam mais exaustão emocional e mais realização pessoal os enfermeiros casados/união de facto, enquanto ao nível da despersonalização são os enfermeiros solteiros/divorciados/viúvos que apresentam valores mais elevados mas sem diferenças estatísticas significativas para os grupos em análise.

Os enfermeiros com o grau de licenciado, manifestam níveis mais elevados de exaustão emocional, despersonalização e realização pessoal e no total do burnout, no entanto, não existem diferenças estatisticamente significativas.

A respeito da despersonalização, encontramos valores médios idênticos nos grupos constituídos a nível da situação profissional, mas sem diferenças estatisticamente significativas. Os enfermeiros com tempo de exercício profissional entre os 10-20 anos são os que apresentam valores mais elevados de exaustão emocional, Os enfermeiros com tempo de serviço inferior a 10 anos (com maior significado nos enfermeiros com menos de 1 ano de tempo no serviço) apresentam valores mais elevados de despersonalização e maior realização pessoal. Nesta análise, apenas a realização pessoal apresentou relevância estatística.

Constatamos valores mais elevados de exaustão emocional e despersonalização, nos enfermeiros que exercem funções numa instituição pública. Os que trabalham em instituições público/privadas encontram-se mais realizados pessoalmente. 
Os enfermeiros que realizam um horário de trabalho fixo, revelam maiores níveis de exaustão emocional e realização pessoal e os que têm um horário rotativo apresentam níveis mais elevados de despersonalização. Não foram encontradas diferenças estatisticamente significativas na variável exaustão emocional. A exaustão emocional e a despersonalização surgem nos enfermeiros que trabalham mais de 40 horas semanais. Os enfermeiros que trabalham menos de 35 horas semanais sentem uma maior realização pessoal.

Quanto à relação entre 0 burnout e o serviço onde os enfermeiros exercem funções, verificamos que são os que trabalham em serviços de obstetrícia e ginecologia os mais exaustos emocionalmente, seguindo-se os que trabalham em cuidados intensivos/urgência/emergência/neonatologia. Os enfermeiros que exercem a sua atividade profissional em cuidados intensivos/urgência/emergência/neonatologia e serviços de saúde mental e psiquiatria apresentam valores mais elevados de despersonalização. Os enfermeiros mais realizados trabalham noutros serviços que não os indicados, seguindo-se os que trabalham em cuidados de saúde primários. Com maiores níveis de burnout, com relevância estatística a nível da despersonalização, encontram-se os enfermeiros que exercem funções em serviços de saúde obstétrica e ginecológica.

No que se refere à relação entre 0 burnout e a realização de turnos suplementares, verificamos que os enfermeiros que realizam turnos suplementares apresentam mais burnout, com médias mais elevadas, de exaustão emocional e despersonalização. São os enfermeiros que não realizam turnos suplementares que apresentam maior realização pessoal.

Quanto à relação entre o burnout e o número de horas acrescido ao horário, são os enfermeiros com um maior acréscimo de horas que apresentam mais burnout, designadamente nas dimensões: exaustão emocional e despersonalização. Os enfermeiros que não têm horas acrescidas são que revelam maior realização pessoal, com diferenças estatisticamente significativas em todas as dimensões.

Na relação entre o burnout e a forma de recompensa pelos turnos suplementares verificamos que são os enfermeiros que assumem que têm outra forma de recompensa que apresentam níveis mais elevados de burnout nas dimensões exaustão emocional e despersonalização. Os enfermeiros cuja recompensa pelos turnos suplementares é monetária revela mais realização pessoal. No entanto, apenas foram encontradas diferenças estatisticamente significativas na exaustão emocional.

Os enfermeiros que assumem ter a capacidade de lidar com conflitos apresentam valores mais elevados de burnout, nas dimensões exaustão emocional e despersonalização. Os enfermeiros que assumem não possuir a capacidade de lidar com conflitos apresentam valores mais elevados de realização pessoal. Existem diferenças estatisticamente significativas em todas as dimensões.

Os enfermeiros que não estão satisfeitos com o serviço onde desempenham a sua atividade profissional apresentam valores mais elevados de exaustão emocional e despersonalização. Por outro lado os que se sentem satisfeitos, apresentam mais realização pessoal.

Relativamente à relação entre o burnout e a satisfação, os enfermeiros que apresentam exaustão emocional e despersonalização encontram-se pouco satisfeitos. Ao nível da realização pessoal, prevalecem os que se manifestam muito satisfeitos. Em todas as dimensões existem diferenças estatisticamente significativas.

Relativamente à associação entre o burnout e as variáveis independentes (variáveis sociodemográficas, socioprofissionais, satisfação e realização e burnout) constatamos que as correlações são classificadas de negativas, à exceção da idade, não se encontrando diferenças estatísticas.

Revelaram-se variáveis preditoras da exaustão emocional: o trabalho, total da satisfação, exaustão pessoal e face ao utente. As variáveis preditoras da despersonalização são a exaustão face ao utente e ao trabalho, idade e sexo. As variáveis preditoras da realização pessoal são 0 burnout pes- 


\section{A SATISFAÇÃO, REALIZAÇÃO E EXAUSTÃO dOS ENFERMEIROS EM PORTUGAL}

soal, burnout face ao utente e burnout face ao trabalho. Apenas a variável burnout face ao trabalho é preditora para o total do burnout (MBI).

\section{DISCUSSÃO}

A velocidade vertiginosa do mundo do trabalho produz muitas vezes a incapacidade de separar o trabalho e a família, ocorrendo situações de exaustão sobretudo, nas profissões de ajuda, como a Enfermagem. 0 burnout enquanto estado de exaustão física, emocional e mental resulta do envolvimento intenso com pessoas, conduzindo a um progressivo sentimento de inadequação e fracasso abrangendo três dimensões: emocional, despersonalização e realização profissional, com particular risco para as pessoas que trabalham com outras (Queirós, 2005).

Ao procuramos saber quais as variáveis sociodemográficas e profissionais que interferem no burnout em enfermeiros a exercer a sua atividade profissional em Portugal, verificamos que a variável sexo interferiu no burnout manifestando as enfermeiras mais exaustão emocional e realização, comparativamente aos enfermeiros. Almeida (2009) também assina-la a inexistência de diferenças significativas na relação do burnout com 0 sexo. Todavia, 0 autor refere que os homens revelam mais despersonalização, tal como no presente estudo, podendo ser justificado com o facto de os homens se envolverem de uma forma diferente no trabalho não envolvendo a família nas questões laborais (Almeida, 2009). Simões (2013), encontrou diferenças significavas nos participantes do sexo masculino, que apresentam valores mais elevados de burnout, contrariamente aos que apuramos no nosso estudo.

Os enfermeiros com idade igual ou superior aos 37 anos são os que revelam mais exaustão emocional, com diferenças estatisticamente significativas. Os enfermeiros com idade igual ou inferior aos 29 anos, apresentam mais despersonalização e os mais velhos valores inferiores, com significância estatística. Os enfermeiros com idade igual ou inferior aos 37 anos, apresentam-se mais realizados profissionalmente. 0 estudo de Miranda (2011) refere que existem diferenças estatisticamente significativas na dimensão exaustão emocional, sendo os enfermeiros mais novos que se encontram mais exaustos comparativamente com os mais velhos. Os resultados de Gomes (2009) afirmam que a relação dos enfermeiros mais novos com as suas expectativas de início de carreira e a adaptação progressiva ao trabalho são indicadores de uma melhor adaptação à frustração, ganhando igualmente mecanismos de defesa, envolvendo-se menos nos contextos de trabalho.

Apresentam mais exaustão emocional e mais realização pessoal os enfermeiros casados/união de facto. A nível da despersonalização e no total do MBI, os enfermeiros solteiros, divorciados e viúvos apresentam valores mais elevados, apesar de a variável estado civil não interferir na síndrome de burnout. Os resultados obtidos não estão em concordância com os de Miranda (2011), porque na dimensão da realização pessoal os profissionais de saúde solteiros revelam mais realização pessoal, em comparação com os casados. 0 autor também verificou que os casados apresentam valores mais elevados de despersonalização, sendo no presente estudo expressivo para os enfermeiros solteiros/divorciados/viúvos.

Os enfermeiros com tempo de exercício profissional entre os 10 e 20 anos são os que apresentam mais exaustão emocional, os com menos de 10 anos de serviço revelam mais realização pessoal e no total do síndrome de burnout assim como na despersonalização.

Miranda (2011), no seu estudo, concluiu que os enfermeiros mais novos apresentam mais despersonalização e mais síndrome de burnout, no total. Assim, os anos de serviço parecem ter relação com o burnout, sendo que os profissionais de saúde com menos experiência profissional são aqueles que revelam maiores médias nas dimensões trabalhar com os utentes, relações profissionais e excesso de trabalho. 
Os enfermeiros que exercem a sua atividade profissional numa instituição pública de saúde apresentam mais exaustão emocional e mais despersonalização. Estão mais realizados pessoalmente, os enfermeiros que trabalham numa instituição público-privada. Neste âmbito, Martins (2010) refere que o tipo de contrato laboral influencia a existência de burnout. Os enfermeiros com horário fixo apresentam mais exaustão emocional e mais realização pessoal.

Os enfermeiros que praticam um horário rotativo apresentam valores mais elevados de despersonalização, tendo esta variável interferido estatisticamente no burnout. Apresentam mais exaustão emocional e despersonalização bem como no total da escala do burnout, os enfermeiros com mais de 40 horas semanais. Os que trabalham menos de 35 horas de trabalho semanal apresentam mais realização pessoal. Na globalidade, os enfermeiros com níveis mais elevados de burnout são os que realizam turnos suplementares e maior número de horas de trabalho acrescido ao horário. Os resultados poderão ser justificados com base no fator excesso de horas de trabalho semanal, o que resulta, como afirma Martins (2010), em maiores níveis de fadiga e stress, como fatores predisponentes a uma maior exaustão emocional e despersonalização.

É importante ressalvarmos que as causas do burnout são multifatoriais, conforme refere Queirós (2005). Trata-se da convergência de características pessoais, do tipo de atividade realizada e da constelação de variáveis provenientes da instituição onde o trabalho é efetuado. Estes fatores podem mediar ou facilitar o processo de stress ocupacional que irá dar lugar ao burnout. As variáveis de personalidade, assim como as sociodemográficas, não são em si deflagradoras da síndrome, mas face a fatores externos relacionados com o trabalho, como o excesso de número de horas, podem facilitar o desencadeamento de burnout. Por outro lado, ainda na opinião do autor, a despersonalização, que constitui uma componente chave das três dimensões do burnout, ao ser uma demostração particular de stress nas profissões assistenciais, pode funcionar como uma estratégia de coping, a que os enfermeiros recorrem para poderem enfrentar a complexidade das situações vivenciadas com o utente e família e mesmo em termos de equipa multidisciplinar e institucional.

Um elevado número de enfermeiros estão sujeitos a uma série de problemas de saúde, nomeadamente perturbações do sono, fadiga excessiva, stress, ansiedade e/ou perturbações sociofamiliares e perturbações orgânicas, devido ao trabalho realizado por turnos rotativos. Os turnos rotativos, a sobrecarga de trabalho e a escassez de profissionais, conduzem para uma sobrecarga física e emocional dos enfermeiros (Ferreira, 2005).

Quanto à relação entre o burnout e o serviço onde exerce funções, verificamos que são os enfermeiros a exercer funções em saúde obstétrica e ginecológica que apresentam valores mais elevados de exaustão emocional, seguidos dos que trabalham em cuidados intensivos, urgência, emergência e neonatologia. Níveis mais elevados de despersonalização são observados nos enfermeiros que trabalham em unidades de cuidados intensivos, urgência, emergência e neonatologia e saúde mental e psiquiatria. Apresentam-se mais realizados os que trabalham noutros serviços, seguindose os que trabalham em cuidados de saúde primários. No total do burnout, apresentam níveis mais elevados os enfermeiros que exercem funções em saúde obstétrica e ginecológica. Martins (2010) contatou que os profissionais de saúde a exercer funções nos serviços de urgências apresentam um valor médio mais elevado de burnout.

Os enfermeiros que assumem ter a capacidade de lidar com conflitos mostram mais exaustão emocional e despersonalização e os que assumem não possuir a capacidade de lidar com conflitos, apresentam mais realização pessoal. 0 facto de os enfermeiros se esforçarem para gerir conflitos poderá levar a valores mais elevados de exaustão emocional e despersonalização.

Apresentam valores elevados de exaustão emocional e despersonalização os enfermeiros que não estão satisfeitos face ao serviço onde trabalham. Refere Gonçalves (2009), que 0 trabalho assu- 


\section{A SATISFAÇÃO, REALIZAÇÃO E EXAUSTÃO dOS ENFERMEIROS EM PORTUGAL}

me-se como uma fonte potencial de motivação sobre as pessoas, as organizações e as outras esferas da vida. Esse potencial é traduzido principalmente por o trabalhador gostar do que faz, transformando 0 trabalho em fonte de realização pessoal, o que justifica o facto de termos verificado que os enfermeiros que se sentem satisfeitos com o local onde trabalham apresentam níveis mais elevados de realização pessoal. Podemos dizer de acordo com Palha (2013), que existem determinadas características que influenciam a forma como a pessoa se sente ou se comporta no seu local de trabalho, sendo a satisfação em relação ao mesmo determinada através de variáveis individuais, organizacionais e ambientais, isto é, resultantes da interação individuo/trabalho que podem ter influência no menor ou maior nível de burnout.

\section{CONCLUSÃO}

Os enfermeiros, considerados trabalhadores expostos a diferentes fatores de stress ocupacional, necessitam adquirir um conjunto de competências que lhes permita manter uma boa saúde mental, evitando situações causadoras de stress que poderão culminar em burnout com prejuízos pessoais, profissionais, sociais e económicos.

Esta síndrome é penalizadora para a saúde mental dos enfermeiros afectando-os, como podemos constatar no presente estudo, em três dimensões: pessoal; trabalho e cliente (Fonte, 2011). Por ser uma doença específica da relação de ajuda, provocando um mal-estar existencial singular e social, coloca o sentido da vida em questão (DelbroucK, 2006).

\section{IMPLICAÇÕES PARA A PRÁTICA CLÍNICA}

É imperativo com base nos resultados obtidos, propor algumas intervenções de modo a reverter esta realidade. Assim, sugerimos a promoção de condições físicas e técnicas nas instituições de saúde; abertura de vagas de ingresso de enfermeiros para diminuição de sobrecarga laboral; desenvolvimento de programas de intervenção sobre gestão do tempo, conflitos, relação interpessoal e comunicação; Estimulo ao reconhecimento do trabalho, valorização e delegação das competências e sistema justo de recompensas dos enfermeiros. Por último esperamos que face aos resultados obtidos e á especificidade do trabalho desenvolvido, seja atribuído um horário semanal de 35 horas aos enfermeiros a exercer funções em todos os serviços de saúde, independentemente do seu vínculo laboral.

\section{REFERÊNCIAS BIBLIOGRÁFICAS}

Almeida, H.F.O. (2009). Comunicação stresse e estratégias de adaptação dos Enfermeiros do Instituto Português de Coimbra de Francisco Gentil EPE (Dissertação de mestrado, Faculdade de Medicina de Coimbra, Portugal).

Conselho Internacional de Enfermeiros. (2011). CIPE: Versão 2: Classificação internacional para a prática de enfermagem. Lisboa: Ordem dos Enfermeiros.

Delbrouck, M. (2006). Síndrome de exaustão: Burnout. Lisboa: Climepsi.

Ferreira, M. M. S. (2005). Risco de saúde dos enfermeiros no local de trabalho. Revista Sinais Vitais, 59, 29-35.

Fonte, C.M.S. (2011). Adaptação e validação para português do questionário de Copenhagen Burnout Inventory CBI (Dissertação de mestrado, Faculdade de Economia da Universidade de Coimbra, Portugal).

Gomes, S.P.S. (2009). Burnout em enfermeiros no início da carreira (Dissertação de mestrado, Universidade de Lisboa Portugal).

Gonçalves, E. M. M. (2009). Satisfação profissional: Uma realidade em cuidados de saúde primários. Enfermagem, 53-54(2), 30-34. 
Martins, J.E.L. (2010). Avaliação processual de burnout em enfermeiros para e na gestão das organizações de saúde (Dissertação de mestrado, Universidade de Trás-os-Montes e Alto Douro, Portugal).

Menezes, M.H.V.F (2010). Novos modelos de gestão hospitalar: Liderança e satisfação profissional em enfermagem (Dissertação de mestrado, Universidade de Trás-os-Montes e Alto Douro, Portugal).

Miranda, S.C.C. (2011). Stress ocupacional burnout e suporte social nos profissionais de saúde mental (Dissertação de mestrado, Faculdade de Filosofia de Braga - Universidade Católica Portuguesa, Portugal).

Palha, M.F.G. (2013). Satisfação profissional dos enfermeiros em unidades de cuidados continuados integrados (Dissertação de mestrado, Instituto Politécnico do Porto, Portugal).

Queirós, P.J.P. (2005). Burnout no trabalho e conjugal em enfermeiros portugueses. Coimbra: Formasau.

Simões, S.C.A. (2013). Burnout em cuidados paliativos (Dissertação de mestrado, Instituto Politécnico de Castelo Branco, Portugal). 
